\title{
Betreuungsrecht: gut gemeint, aber mangelhaft realisiert - Kontra
}

\author{
Guardianship Law: Well Meant, but Deficiently Realized - Contra
}

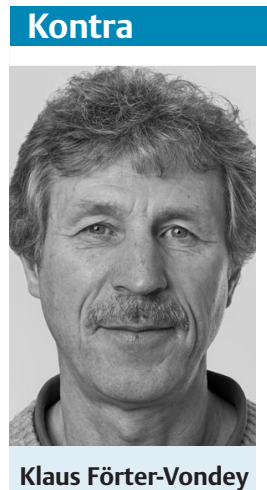

Immerhin, das Betreuungsrecht (BtG), wird als sinnvoll angesehen. Wenn mit mangelhafter Realisierung die Betreuungspraxis gemeint ist, ist die Bewertung bitter. Nun lässt sich aus Sicht der Betreuungspraxis Ähnliches über die Psychiatrie und die psychiatrische Praxis feststellen: Psychiatrie ist sinnvoll, die Ausführung häufig mangelhaft. Es bei dieser gegenseitigen, wenig differenzierten Beurteilung zu belassen, wäre aber nicht nur falsch, sondern auch fahrlässig den Menschen gegenüber, die zu einem hohen Prozentsatz sowohl auf Betreuung als auch auf eine psychiatrische Versorgung angewiesen sind und häufig auch auf deren $\mathrm{Zu}$ sammenarbeit. Deswegen sollte es um Differenzierung und gemeinsamer Problembearbeitung und nicht um gegenseitige Verurteilung gehen. Wissen bietet sich in der Regel als Grundlage für Verständnis und Zusammenarbeit an. Dabei gehe ich davon aus, dass die Aufgabenstellung der Psychiatrie mehr bekannt ist als die der Betreuung.

\section{Betreuungsrecht sinnvoll}

Wie sollte es anders sein: Auch wir halten das BtG für sinnvoll, obwohl wir gerade vor dem Hintergrund der Behindertenrechtskonvention (BRK) einen erheblichen Reformbedarf feststellen. Bleiben wir aber beim gültigen Recht.

Sinnvoll ist, dass ein „Volljähriger (der) aufgrund einer psychischen Krankheit oder einer körperlichen, geistigen oder seelischen Behinderung seine Angelegenheiten ganz oder teilweise nicht besorgen (§1896 BGB)“" kann, durch die Betreuung eine Unterstützung erhält.

Die große Schnittmenge von zu psychiatrischen PatientInnen und BetreuungsklientInnen erfordert eine Arbeitsbezie- hung zwischen Psychiatrie und Betreuung, die häufig durch gegenseitige unerfüllbare Erwartungen belastet wird. Im Betreuungsrecht (BtG) heißt es zur Aufgabenstellung: „Der Betreuer hat die Angelegenheiten des Betreuten so zu besorgen, wie es dessen Wohl entspricht. Zum Wohl (...) gehört auch die Möglichkeit, im Rahmen seiner Fähigkeiten sein Leben nach seinen eigenen Wünschen und Vorstellungen zu gestalten. (...) Der Betreuer hat Wünschen des Betreuten zu entsprechen, soweit dies dessen Wohl nicht zuwiderläuft (...). Der Betreuer (hat) dazu beizutragen, dass Möglichkeiten genutzt werden, die Krankheit oder Behinderung des Betreuten zu beseitigen, zu bessern, ihre Verschlimmerung zu verhüten oder ihre Folgen zu mildern ( $§ 1901,2$ und 3 BGB).“ Angelegenheiten, so definieren wir aus fachlicher Sicht, sind wunsch- und wohlbegründete Anliegen und Probleme, die von der KlientIn nicht mehr erkannt oder deren Sinn ganz oder teilweise nicht verstanden wird und keine entsprechende Handlungs- und Durchsetzungsmöglichkeit besteht.

Wir stehen also nur auf der subjektiven Seite der KlientInnen. Wir nennen unsere Tätigkeit Besorgungsleistung. Damit erhalten KlientInnen eine jeweils erforderliche Zurüstung zum Selbstmanagement und zur Selbstverantwortung unter Berücksichtigung des Willens (Lebensentwurf) und des Wohls. Dieser Prozess ist gemeinsame „Produktion von Wohlfahrt“. Das ist einer medizinischen Behandlung ähnlich. Der Arzt ist auf die Mitwirkung des Patienten angewiesen. Die Besorgungsleistung erfolgt in Abgrenzung zur Versorgungsleistung (klar definierten Aufträge z.B. in der Pflege, pädagogischen Hilfen etc.). Die Besorgung bezieht sich ggf. auf alle Lebensbereiche, also nicht nur auf die Behandlung.

Würde Betreuung allein als Instrument verstanden z.B. zur Durchsetzung eines ärztlichen Behandlungsvorschlags auch gegen den Willen des Klienten, muss das zu Differenzen in der Arbeitsbeziehung führen. Sinnvoll wäre eine Darstellung der Notwendigkeit der Behandlung. Wir sind dann in der Lage im Austausch mit dem Klienten zum Selbstmanagement und zur Selbstverantwortung ganz oder teilweise zuzurüsten, um zu einer akzeptierten Behandlung zu kommen. Das ist der Sinn von Betreuung.

\section{Ausführung mangelhaft}

Damit zur Betreuungspraxis, die wie in der Überschrift signalisiert, als mangelhaft empfunden wird. Betreuungspraxis ist die Leistung, die durch die BetreuerInnen (nicht von Gerichten und Behörden) erbracht wird. Ein Vergleich: Würde von einer psychiatrischen Versorgung erwartet, dass der Patient auch gepflegt oder seine Wohnung gereinigt wird, was dann aber nicht erfolgt, geräte diese in ein schlechtes Licht, weil die Erwartung nicht erfüllt würde.

Ähnlich verhält es sich mit der Betreuung. Beispiel: Der Nachbar ist zu laut. Es wird erwartet, dass er „entmündigt“ und ins Heim gebracht wird. Es besteht gleichzeitig die „negative Erwartung“, also Angst vor einer „Entmündigung“ durch die Betreuung (Entmündigung ist seit 1992 nicht mehr möglich!). Oder: Die Vorstellung des Klienten beim Psychiater wird als Pflicht verstanden. Diese Erwartungen kann nicht erfüllt werden, obwohl aus fachlicher Sicht die erbrachte Betreuungsleistung gut ist.

Die Aufgabe der Betreuung besteht darin, die Wünsche der Menschen unter Beachtung ihres Wohls umzusetzen und nicht, die Erwartungen Dritter zu erfüllen. Wir nennen Betreuung deswegen Be- und nicht Entrechtung. Von daher kann die Beurteilung der Betreuungspraxis als mangelhaft u.U. auf der Grundlage falscher Erwartungen und eines falschen Verständnisses vom Sinn des Gesetzes erfolgt sein.

Zugegebenermaßen liegt die Beurteilung der Betreuung nicht allein an dem Missverständnis gegenüber dem BtG und der Praxis. Es gibt mindestens zwei weitere 
Faktoren, die sich im Prinzip auch in der psychiatrischen Praxis finden lassen: Die Berufsausübung erfolgt unterschiedlich und die Rahmenbedingungen begrenzen die Qualität.

Ich bleibe bei den Rahmenbedingungen, da die Betrachtung einer individuellen Berufsausübung in allen Berufen zumindest nur zum Teil als prinzipielles Problem betrachtet werden kann.

Die Notwendigkeit der Anerkennung einer professionellen Betreuung wird mittlerweile fast nur noch von der Regierung bestritten. Entwickelt ist ein Verfahren (Betreuungsmanagement). Kenntnisse in vielen Bereichen - auch medizinische - sind vorzuhalten. Nach 20 Jahren BtG ist das in einer Ausbildung (Master) zu gewährleisten. Die geforderte Qualität und eine unabhängige Unterstützung und Vertretung von KlientInnen ist nur durch berufliche Unabhängigkeit und in Selbstverwaltung zu sichern. Die Politik verweigert diese Grundlagen. Und dabei verfügen fast alle Berufsinhaber in der Regel über Hochschulabschlüsse und über Lebens- und Berufserfahrung.

Die materielle Absicherung der Berufsinhaber ist desaströs. Erwartungen an die Betreuung sind häufig auch wegen der Zeitbemessung pro Fall (Pauschalen) von 3,2 Stunden im Schnitt pro Monat pro Klient nicht erfüllbar. Darin enthalten sind Zeiten für die aufsuchende Arbeit (Wege zum Klienten, zum Arzt usw.), Durchsetzung von Ansprüchen gegenüber Verwaltung und Versicherung (immer mehr Klagen). Deswegen kollidiert z.B. eine sinnvolle Begleitung in die psychiatrische Praxis (Sicherung des Zugangs zur Behandlung) mit einer Wartezeit dort von 1-2 Stunden, plus An- und Abreise mit der wirtschaftlichen Zumutbarkeit. Denn auch über die Stundensätze von (in der höchsten Stufe) 44 Euro pro Stunde ist keine Kompensation der geringen Zeitkontingente möglich.

Den Gesetzgeber - um auf das Betreuungsrecht zurückzukommen - interessiert das wenig. Ausbildung, Qualitätssicherung und berufliche Selbstverwaltung, so wird befürchtet, trägt zur Kostensteigerung bei. Spätestens im Zusammenhang mit der Anerkennung der Behindertenrechtskonvention als geltendes Recht halten wir dieses Umgehen für skandalös.

\section{Fazit}

Das sinnvolle Betreuungsrecht, durch das der Volljährige, der seine Angelegenheiten nicht besorgen kann, eine Unterstützung in Form einer Betreuung erhält, gerät vor dem Hintergrund der Rahmenbedingung und eines Reformstaus immer mehr unter Druck. Dem BtG und der Praxis droht damit eine Sinnentleerung. Die Bewertung der Praxis als mangelhaft ist häufig mit Erwartungen konfrontiert, die prinzipiell nicht erfüllbar sind. Und es bieten z.B. nicht vorhandene Zugangsvorausetzung zum Beruf und eine fehlende Qualitätssicherung vielfältige Möglichkeiten für die Berufsausübung.
Es ist auch darauf zu verweisen, dass immer mehr die Psychiatrie betreffende Regelungen in das BtG geschrieben werden und $\mathrm{zu}$ weiteren Belastungen/Erwartungen beitragen. Ich nenne hier nur die Patientenverfügung und die Verpflichtungen im Zusammenhang bzgl. der Zwangsmedikation. Betrachten wir auch noch die stationäre und ambulante psychiatrische Versorgungssituation, ergibt sich eine schwierige Situation für PatientInnen und KlientInnen, Psychiatrie und Betreuung, sodass sich aus meiner Sicht deutlich eher gemeinsame Kampffelder entdecken lassen als mehr oder weniger schmeichelhafte Zuweisungen von Qualitätsprädikaten.

Sie haben eine eigene Meinung zu diesem Thema? Dann schreiben Sie uns an: psychiat-praxis@thieme.de!

\section{Korrespondenzadresse \\ Klaus Förter-Vondey}

Beratung und Betreuung

Roder \& Förter-Vondey GbR

Steindamm 91

20099 Hamburg

foerter-vondey@beratung-und-betreuung.de

Bibliografie

DOI http://dx.doi.org/

10.1055/s-0033-1359945

Psychiat Prax 2014; 41: 69-70

(c) Georg Thieme Verlag KG

Stuttgart · New York

ISSN 0303-4259 